\title{
Early Radiographic Response to Epidermal Growth Factor Receptor-Tyrosine Kinase Inhibitor in Non-small Cell Lung Cancer Patients with Epidermal Growth Factor Receptor Mutations: A Prospective Study
}

\author{
John WC Chang ${ }^{1}$, Ming-Mo Hou ${ }^{1}$, Jia-Juan Hsieh ${ }^{1}$, Yun-Chung Cheung ${ }^{2}$, Hung-Ming Wang ${ }^{1}$, \\ Jen-Shi Chen ${ }^{1}$, Cheng-Hsu Wang ${ }^{1}$, Chih-Hung Chen ${ }^{3}$, Kun-Yun Yeh ${ }^{1}$, Li-Ying Ou ${ }^{1}$, Chia-Hsun Hsieh ${ }^{1}$, \\ Hong-Dar Isaac $\mathrm{Wu}^{4}$, Ying-Tsong Chen ${ }^{5,6}$, Il-Chi Chang ${ }^{5}$, Shiu-Feng Huang ${ }^{5,7,8}$
}

Background: The time schedules for response evaluation of epidermal growth factor receptor-tyrosine kinase Inhibitor (EGFR-TKI) in non-small cell lung cancer (NSCLC) patients are still ill-defined.

Methods: $\quad$ Stage IIIB/IV patients with histologically proven NSCLC were enrolled in this study if the tumor cells bore EGFR mutations other than T790M. Eligible patients were treated with either $250 \mathrm{mg}$ of gefitinib or $150 \mathrm{mg}$ of erlotinib once daily. The early response rate [computed tomography (CT) scan on Day 14], definitive response rate determined on Day 56, progression-free survival (PFS), overall survival (OS), and toxicity profile were assessed prospectively.

Results: $\quad$ Thirty-nine patients were enrolled in this study. A total of 29 patients $(29 / 39,74.4 \%)$ achieved partial response (PR). Twenty-one patients $(21 / 39,53.8 \%)$ had early radiological response on Day 14 . The early radiological response rate in patients with PR was $72.4 \%$ (21/29). Only eight patients without a PR on early CT still ended with PR. Among the 29 patients with PR, the PFS (8.1 months) and OS (18.3 months) of the 21 patients with early CT response were shorter than those of the 8 patients without early CT response (11.9 and 24.0 months for PFS and OS, respectively). But the survival differences were statistically non-significant.

Conclusions: A very high percentage $(72.4 \%, 21 / 29)$ of NSCLC patients with EGFR mutations with PR demonstrates early radiological response to EGFR-TKIs, which would advocate early radiological examination for EGFR-TKI therapy in NSCLC patients.

(Biomed J 2015;38:221-228)

Key words: computed tomography scan, epidermal growth factor receptor, non-small cell lung cancer,

treatment response, tyrosine kinase inhibitor

\section{At a Glance Commentary}

Scientific background of the subject

The time schedules for response evaluation of epidermal growth factor receptor-tyrosine kinase inhibitor (EGFR-TKI) in non-small cell lung cancer (NSCLC) patients are still ill-defined. In this prospective study, we attempted to determine the TKI response, including the early radiological response rate (on Day 14), overall response rate (ORR), PFS, and overall survival (OS) to EGFR-TKI treatment in NSCLC patients bearing EGFR mutations.

\section{What this study adds to the field}

A very high percentage $(72.4 \%, 21 / 29)$ of NSCLC patients with EGFR mutations with PR demonstrated early radiological response to EGFR-TKIs, which would advocate early radiological examination for EGFR-TKI therapy in NSCLC patients.

\begin{abstract}
${ }^{1}$ From the Division of Hematology-Oncology, Department of Internal Medicine, Chang Gung Memorial Hospital at Linkou, Chang Gung University College of Medicine, Taoyuan, Taiwan; ${ }^{2}$ Department of Diagnostic Radiology, Chang Gung Memorial Hospital at Linkou, Chang Gung University College of Medicine, Taoyuan, Taiwan; ${ }^{3}$ Division of Pulmonology, Department of Internal Medicine, Chang Gung Memorial Hospital at Linkou, Chang Gung University College of Medicine, Taoyuan, Taiwan; ${ }^{4}$ Department of Applied Mathematics and Institute of Statistics, National Chung-Hsing University, Taichung, Taiwan; ${ }^{5}$ Institute of Molecular and Genomic Medicine, National Health Research Institutes, Zhunan, Taiwan; ${ }^{6}$ Institute of Genomics and Bioinformatics, National Chung-Hsing University, Taichung, Taiwan; ${ }^{7}$ Department of Pathology, Chang Gung Memorial Hospital at Linkou, Chang Gung University College of Medicine, Taoyuan, Taiwan; ${ }^{8}$ Department of Pathology, Tzu-Chi Medical Center, Taipei Branch, Tzu-Chi University, Taipei, Taiwan Received: Dec. 2, 2013; Accepted: Jul. 20, 2014

Correspondence to: Dr. Shiu-Feng Huang, Division of Molecular and Genomic Medicine, National Health Research Institutes, Taiwan. 35 Keyan Road, Zhunan, Miaoli 35053, Taiwan. Tel: 886-37-246166 ext. 35315; Fax: 886-37-586459; E-mail: sfhuang@ nhri.org.tw
\end{abstract}

DOI: $10.4103 / 2319-4170.138320$ 
L ung cancer, especially non-small cell lung cancer (NSCLC), has become the leading cause of cancer deaths in most parts of the world due to its high mortality rate. ${ }^{[1,2]}$ Thus, it has also become the leading target for the development of new anti-cancer agents. Among the targeted therapies that inhibit activated protein kinases with small-molecule drugs, epidermal growth factor receptor-tyrosine kinase inhibitors (EGFR-TKIs) have been active in the treatment of advanced NSCLC. Two drugs, erlotinib (Tarceva) and gefitinib (Iressa; AstraZeneca Inc., Menchester, UK), have been shown to have survival benefit in Caucasians and Asians, respectively, when compared to placebo in controlled, double-blinded, randomized phase III trials. ${ }^{[3,4]}$ These two EGFR-TKIs disrupt EGFR signaling by competing with adenosine triphosphate (ATP) for the binding sites at tyrosine kinase domain, and result in inhibition of phosphorylation and the downstream signaling network. These EGFR-TKIs are only effective to a subgroup of NSCLC patients and act faster than conventional chemotherapy, but have relatively mild side effects. ${ }^{[5,6]}$ In 2004, Lynch et al. and Paez et al. identified mutations in the tyrosine kinase domain of EGFR gene, which were correlated with the clinical responsiveness to gefitinib. ${ }^{[7,8]}$ We reported a very high $E G F R$ mutation rate in the NSCLC patients in Taiwan, mainly with adenocarcinoma, and its association with the clinical responsiveness (the first report from Asian countries) in 2004, ${ }^{[9]}$ soon after the above two reports from Harvard Medical School. Various reports from other Asian countries have shown similar results. ${ }^{[10-12]}$ Three prospective studies from Japan demonstrated that the overall response rate (ORR) in patients who have mutated NSCLC and received TKI treatment was $75 \%$, and the median progression-free survival (PFS) was 8.9-11.5 months. ${ }^{[13-15]}$ Yang et al. also reported a longer time of progression to TTF (time to treatment failure) in NSCLC patients bearing exon 19 deletion or L858R mutation of EGFR than the wild type in advanced NSCLC patients receiving first-line gefitinib monotherapy. ${ }^{[16]}$ In contrast, among unselected NSCLC patients, the objective response rate was only about $10 \% .^{[5,6]}$ Thus, EGFR mutations have become an important molecular biomarker for physicians to choose an appropriate first-line treatment for NSCLC patients. ${ }^{[17,18]}$

Conventionally, the response to anticancer treatment, including targeted therapy, is evaluated after the patient completes the second course of treatment. ${ }^{[17]}$ Although the response to EGFR-TKI could be dramatic in a few days, the actual timing to reach a radiographic response has not been well studied. The time schedules for response evaluation are also still ill-defined. In order to predict the early responsiveness to gefitinib, Sunaga et al. evaluated the changes in ${ }^{18} \mathrm{~F}$-fluorodeoxyglucose (FDG) uptake by positron emission tomography (PET) on Day 2 and Day 28 after the initiation of gefitinib therapy in five patients with NSCLC. ${ }^{[19]}$ In patients who eventually achieved radiographic partial response (PR) and stable disease (SD), the uptake of FDG decreased up to $61 \%$ and $59 \%$, respectively, on Day 2 and $26 \%$ and $43 \%$, respectively, on Day 28. In contrast, FDG uptake was increased up to $153 \%$ on Day 2 and $232 \%$ on Day 28 in one patient with progressive disease (PD). Also, in a xenograft model, Ullrich et al. demonstrated that erlotinib-sensitive tumors exhibited a striking and reproducible decrease in $3^{\prime}$-deoxy- $3^{\prime}$ - $\left[{ }^{18} \mathrm{~F}\right]$-fluoro-l-thymidine $\left({ }^{18} \mathrm{~F}\right.$-FLT) uptake after only 2 days of treatment. ${ }^{[20]}$ The above preliminary results suggested that PET may be useful in predicting EGFR-TKI response in very early days. However, a recent study has demonstrated that it is the radiographic response, rather than the PET scan response, that predicted survival after neoadjuvant chemotherapy for resectable NSCLC. ${ }^{[21]}$ The role of PET in evaluating the treatment outcome is yet to be determined. Consequently, defining the early response of patients with NSCLC by computed tomography (CT) scan examination remains a very important issue.

In this prospective study that was started in 2005 , we attempted to determine the TKI response, including the early radiological response rate (on Day 14), ORR, PFS, and overall survival (OS) to EGFR-TKI treatment in NSCLC patients bearing EGFR mutations. Radiological response to EGFR-TKIs was found on Day 14 in 21 of 39 patients $(53.8 \%)$ and on Day 56 in 21 of 29 patients $(72.4 \%)$ with confirmed PR. This may be the first report regarding the correlation between the early radiological image change and the overall response to EGFR-TKI in NSCLC patients with EGFR mutations.

\section{PATIENTS AND METHODS}

\section{Phase II clinical trial design}

This single-center, phase II study was approved by the Institutional Review Board (IRB) of the Chang Gung Memorial Hospital (CGMH) in 2005 (protocol No. CMRPG: 350031, IRB No. 94-0615B and No. 96-0086C), and written informed consent was obtained from all enrolled patients. The primary objective of this study was to determine the treatment response to EGFR-TKIs on Day 56 and the early radiological response (by CT scan on Day 14) in NSCLC patients with EGFR mutations and stage IIIB/stage IV disease. Secondary objectives were to correlate the TKI response with different $E G F R$ mutations and to evaluate the safety and efficacy (PFS and OS) of TKI treatment.

The inclusion criteria were: Patients of age 18 years or older, having confirmed non-squamous NSCLC histology, positive for EGFR mutation other than T790M (the mutation analyses need to done on fresh tumor tissue samples only), not amenable to curative surgery or radiotherapy, chemotherapy-naïve or failure of prior treatment with one chemotherapy regimen (platinum-based regimen), Eastern 
Cooperative Oncology Group performance status of 0-3 and an estimated life expectancy of more than 12 weeks, fully recovered from toxic effects of previous antitumor therapy, not having taken chemotherapy within 1 month, and with adequately functioning liver [total bilirubin $<1.25$ times the upper normal limit (UNL) of the institutional normal value, transaminases $<5$ $\times \mathrm{UNL}$, alkaline phosphatase $<6 \times \mathrm{UNL}]$, kidneys (serum creatinine $<1.5 \times \mathrm{UNL}$ ), and bone marrow (hemoglobin $\geqq 10 \mathrm{~g} / \mathrm{dl}$, neutrophils $\geqq 2000 / \mu l$, platelets $\geqq 100,000 / \mu l$ ). The postoperative recurrent NSCLC could also be included. Brain metastasis could be included only if no symptoms were present.

The exclusion criteria were: Central nervous system (CNS) metastasis unless the patients were clinically stable 6 weeks after radiotherapy, secondary malignancies, and major systemic diseases. The eligible patients received erlotinib $150 \mathrm{mg} /$ day or gefitinib $250 \mathrm{mg} /$ day after signing the informed consent and completing the screening procedures. $\mathrm{CT}$ scan of all of the measurable tumor sites was performed on Day 14, Day 56, and then every 8 weeks, and the lesions were evaluated using Response Evaluation Criteria in Solid Tumor (RECIST). ${ }^{[22]}$ The time point for determination of treatment response was on Day 56. Treatment was continued until disease progression as documented by imaging studies or until development of unacceptable toxicity. Adverse events were recorded every 2 weeks according to the National Cancer Institute Common Toxicity Criteria version 3. If the patients had documented treatment failure to EGFR-TKIs, they were withdrawn from this study and were advised to receive chemotherapy after the discontinuation of EGFR-TKIs.

\section{Tissue acquisition and EGFR gene mutation analysis}

Most of the tumor tissues for mutation analyses $(34 / 39,87.2 \%)$ were fresh-frozen tissues acquired from CT-guided core needle biopsy or from wedge resection of lung or craniotomy for brain metastasis at the time of diagnosis. Two samples were collected from pleural effusion and three samples were formalin-fixed paraffin-embedded tissues. For the mutational analysis of the kinase domain of $E G F R$, coding sequences from exons 18 to 21 were amplified by polymerase chain reaction (PCR) and subjected to direct sequencing as previously described. ${ }^{[9,23]}$ Sequencing reactions was electrophoresed on an ABI 3700 genetic analyzer. Chromatography was reviewed by two investigators with manual and BLAST software with the EGFR reference sequence (NM_005228.3, NCBI). All sequence variations were re-examined by a second independent PCR amplification and repeated sequencing reactions.

\section{Statistical analyses}

The PFS and OS were estimated by the Kaplan-Meier method. The log-rank test was used to assess between-group differences. A two-sided $p<0.05$ was considered statistically significant. The response and toxicity data were analyzed using simple descriptive statistics.

\section{RESULTS}

\section{Patient characteristics and $E G F R$ gene mutations}

From October 2005 to December 2008, 112 NSCLC patients of non-squamous histology had the tumor samples available for EGFR mutation analyses. Sixty patients (54\%) were EGFR mutation positive. Patients with T790M mutations were not included. Among the 60 patients, 39 were enrolled in this study; the remaining 21 patients were either not eligible or underwent chemotherapy [Figure 1]. All patients were Taiwanese and the median age was 62 years (range 41-79). The clinicopathologic characteristics are listed in Table 1. The pathological diagnoses for all samples were adenocarcinoma. The EGFR mutations identified were mainly exon 19 deletions (15 patients) and L858R point mutations (21 patients).

\section{TKI therapeutic response evaluated by CT scan}

Thirty-four patients received gefitinib $250 \mathrm{mg}$ daily and five patients received erlotinib $150 \mathrm{mg}$ daily. In two of these five patients, erlotinib was the second targeted therapy after they developed drug resistance to gefitinib. One of these two patients has been reported previously. ${ }^{[24]}$ All 39 patients were fully assessable for efficacy and toxicity. The response patterns and survival data are shown in Table 2. The median follow-up time was 24.0 months (range 9.5-47.5 months). Twenty-nine (74.4\%) of the $39 \mathrm{pa}-$ tients achieved PR, 6 (15.4\%) had SD, and $4(10.2 \%)$ had PD. The median PFS was 6.8 months [95\% confidence interval (CI): 4.3-9.5 months]. The median OS was 15.9 months (95\% CI: 6.6-25.2 months). A total of 21 (53.8\%) of the 39 patients demonstrated early radiographic response by CT scan performed on Day 14 [Figure 2]. The early radiological response rate in patients with PR was $72.4 \%(21 / 29)$. Only eight patients without a PR on the early CT still ended with PR.

\section{TKI therapeutic response and survival}

For patients with PR confirmed by the CT scan on Day 56, but not shown on Day 14, the median PFS was 11.9 months (95\% CI: 9.6-14.2 months). For patients with PR detected on Day 14, the median PFS was 8.1 months (95\% CI: 6.0-11.9 months). The PFS showed no significant differences between patients with and without early response $(p=0.4059)$. The median PFS for patients with SD and PD were 4.9 months and 2.1 months, respectively. The overall log-rank test for PFS, when comparing the four 


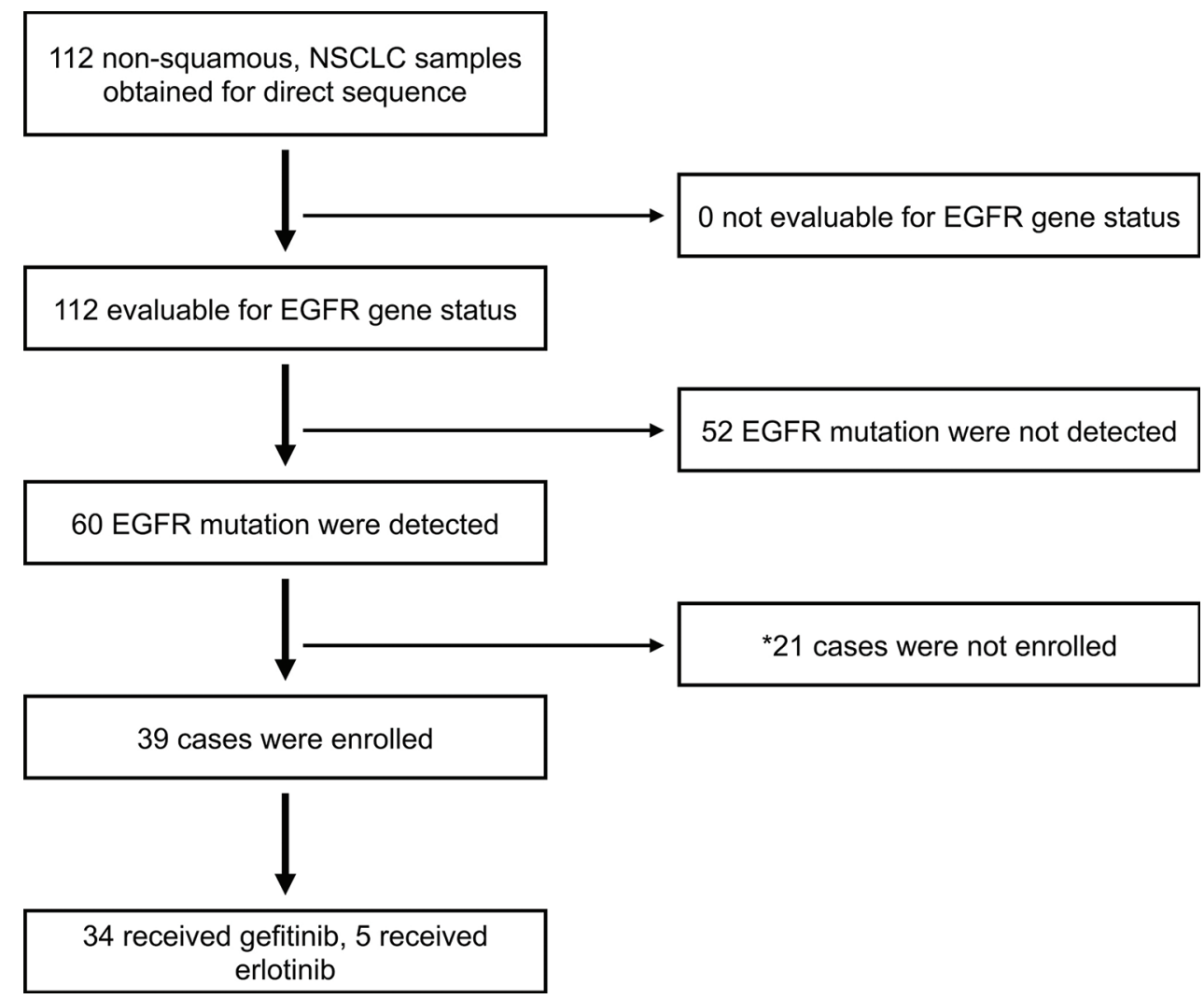

Figure 1: The schema of patient selection is presented in a consort diagram. Initially, we analyzed 112 consecutive non-squamous NSCLC samples for the DNA sequence, and all the samples were evaluable for EGFR mutation. Sixty samples were EGFR mutation positive other than T790M. A total of 39 patients were enrolled in the study. Thirty-four patients received gefitinib and five patients received erlotinib. *Twenty-one patients were either not eligible or received chemotherapy.

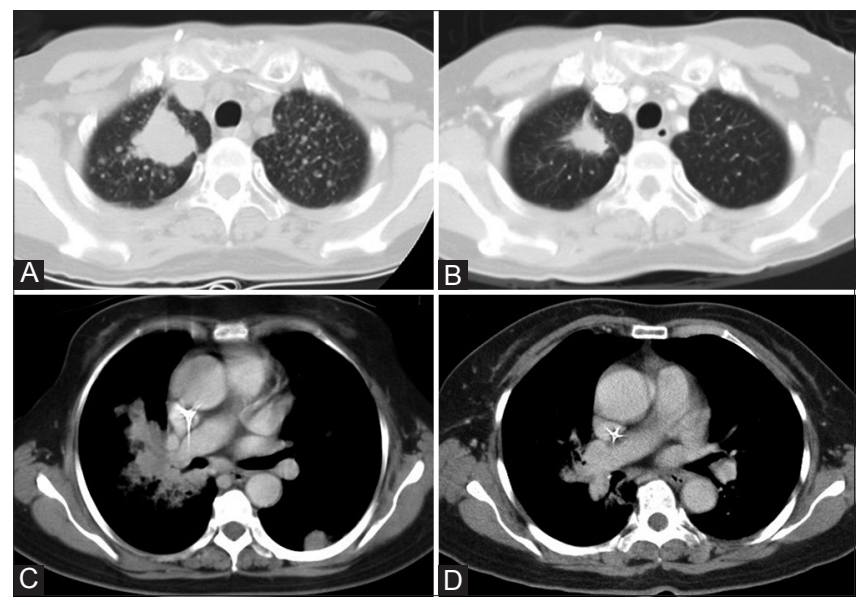

Figure 2: Early responses to EGFR-TKI were determined by CT scan performed on Day 14. (A and C) The CT scan images before EGFRTKI treatment for Patient 1 and Patient 16, respectively. (B and D) Marked tumor shrinkage was found after EGFR-TKI treatment for 2 weeks in Patient 1 and Patient 16, respectively.

groups of patients (PR, early PR, SD, and PD), showed a significant difference $(p<0.0001)$ [Figure 3A]. For patients with PR confirmed by CT scan on Day 56, but not shown on Day 14, the median OS was 24.0 months (95\% CI: 22.0 months).
For patients with PR detected on Day 14, the median OS was 18.3 months (95\% CI: 9.9-28.1 months). Also, there was no significant difference in the OS between patients with and without early response $(p=0.3999)$. The median OS for patients with SD was 9.8 months (95\% CI: 6.8-12.8 months). The median OS for patients with PD had not reached yet due to the small number of cases (two died and two still alive). The overall log-rank test for OS, when comparing the four groups of patients (PR, early PR, SD, and PD), also showed significant difference ( $p=0.0186$ ) [Figure 3B]. There was no significant difference in the OS between patients with PR determined on Day 56, and PD was mainly due to the small number of cases in the PD group.

Among the 39 patients, 27 receiving gefitinib as the first-line treatment had similar efficacy. The median PFS was 8.1 months (95\% CI: 5.9-10.2 months) and the median OS was 15.9 months (95\% CI: 9.9-21.9 months). Twenty-one (77.8\%) patients achieved PR on Day 56 and 14 (72.4\%) of the 21 patients with PR achieved early response on Day 14.

\section{EGFR mutation subtypes and survival}

We also analyzed the relationship between the efficacy and subtypes of EGFR mutation. The early 


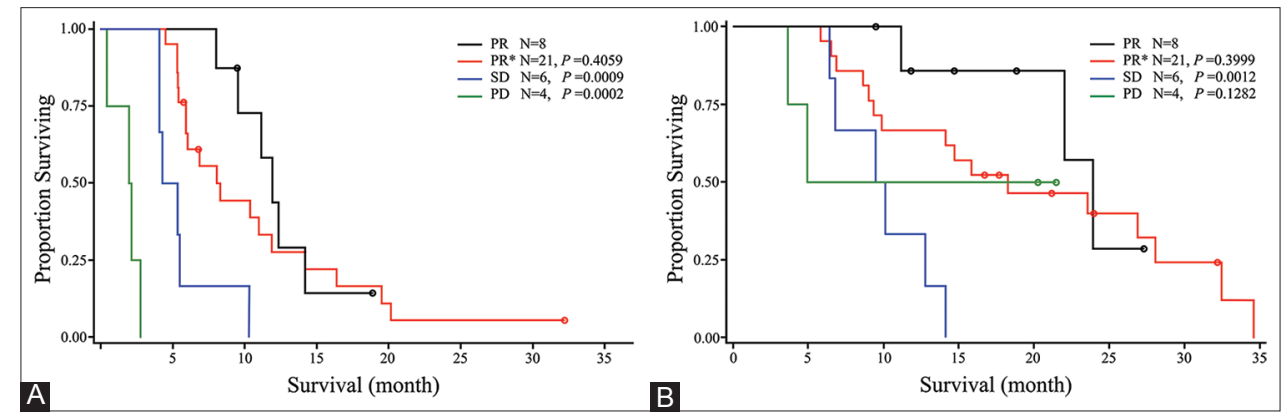

Figure 3: The 39 lung adenocarcinoma patients were divided into four groups according to their response patterns: PR, patients with partial response confirmed by the CT scan on Day 56, but not shown on Day 14; PR*, patients with early partial response confirmed by CT scan on Day 14; SD, patients with stable disease; and PD, patients with progressive disease. (A) Kaplan-Meier curves for progression-free survival (PFS) of the PR group versus the other three groups ( $\mathrm{PR}^{*}, \mathrm{SD}$, and PD, respectively). The median PFS of the four groups were: PR $=11.9$ months, $\mathrm{PR}^{*}=8.1$ months, $\mathrm{SD}=4.9$ months, and $\mathrm{PD}=2.1$ months. Of the patients with $\mathrm{PR}$, there were no significant differences between patients with and without early response $(p=0.4059)$. (B) Kaplan-Meier curves for overall survival (OS) of the PR group versus the other three groups (PR*, SD, and PD, respectively). The median survival of the four groups was: $\mathrm{PR}=24.0$ months, $\mathrm{PR} *=18.3$ months, $\mathrm{SD}=9.8$ months, and $\mathrm{PD}=$ not reached yet (two died and two were alive).

Table 1: The clinicopathologic characteristics of 39 NSCLC patients

\begin{tabular}{|c|c|c|}
\hline Variables & Number & $\%$ \\
\hline Patients enrolled & 39 & 100 \\
\hline \multicolumn{3}{|l|}{ Gender } \\
\hline Male & 16 & 41 \\
\hline Female & 23 & 59 \\
\hline \multicolumn{3}{|l|}{ Age (years) } \\
\hline Median (range) & $62(41-79)$ & \\
\hline \multicolumn{3}{|l|}{ Stage } \\
\hline IIIB & 3 & 8 \\
\hline IV & 36 & 92 \\
\hline \multicolumn{3}{|l|}{ Pathology diagnosis } \\
\hline Adenocarcinoma & 39 & 100 \\
\hline \multicolumn{3}{|c|}{ Site of distant metastasis } \\
\hline Lung & 15 & 39 \\
\hline Liver & 8 & 21 \\
\hline Bone & 17 & 44 \\
\hline Brain & 13 & 33 \\
\hline \multicolumn{3}{|l|}{ ECOG PS } \\
\hline 0 & 2 & 5 \\
\hline 1 & 21 & 54 \\
\hline 2 & 16 & 41 \\
\hline \multicolumn{3}{|c|}{ Number of prior chemotherapies } \\
\hline$>2$ & 2 & 5 \\
\hline $1-2$ & 10 & 26 \\
\hline 0 & 27 & 69 \\
\hline \multicolumn{3}{|c|}{ Previous targeted therapy } \\
\hline Yes & 2 & 5 \\
\hline No & 37 & 95 \\
\hline
\end{tabular}

Abbreviations: NSCLC: Non-small cell lung cancer; ECOG PS:

Eastern cooperative oncology group performance status

response rates were $60 \%(9 / 15)$ in patients with exon 19 deletion and $52.4 \%(11 / 21)$ in patients bearing L858R. The ORRs were $80 \%(12 / 15)$ in patients with exon 19 deletion and $71.4 \%(15 / 21)$ in patients bearing
L858R. The PFS and OS of patients with exon 19 deletion were 5.9 months (95\% CI: 3.8-8 months) and 18.3 months (95\% CI: 9.6-27 months), respectively. In the group of patients bearing L858R, the PFS and OS were 8.1 months $(95 \% \mathrm{CI}$ : 5-11 months) and 12.8 months (95\% CI: 7-18.5 months), respectively. There were no significant differences in these two groups in terms of PFS $(p=0.82)$ and OS $(p=0.858)$.

\section{Safety}

Treatment-related adverse effects (AEs) are summarized in Table 3. The most frequent AEs seen in this study were skin rash (46.2\%), dry skin (38.5\%), diarrhea (30.7\%), and anemia (41\%). Grade 3 or 4 paronychia, skin rash, and diarrhea were seen in $5.1 \%, 7.7 \%$, and $2.6 \%$ of the patients, respectively. Three patients required dose reduction to $250 \mathrm{mg}$ of gefitinib every other day because of toxicity (one with liver toxicity, one with skin toxicity, and one with paronychia). No life-threatening AEs, such as interstitial lung disease (ILD), were observed in this study. The complication rate of CT-guided core needle biopsy was $12.8 \%$ for pneumothorax and $6.4 \%$ for hemoptysis. There was no procedure-related mortality.

\section{DISCUSSION}

It was well demonstrated in Iressa Pan-Asia Study (IPASS) trial and a recent report from Japan that gefitinib was active mainly in EGFR-mutated NSCLC. ${ }^{[25,26]}$ Therefore, it is very important to pinpoint the $E G F R$ status by applying sensitive techniques to adequate tumor samples. In all the prospective studies published previously, the efficacy of EGFR-TKI in EGFR-mutated NSCLC patents was quite good. The response rate varied from $75 \%$ to $91 \%$, and the PFS from 7.7 to 12.9 months. ${ }^{[13,14,19,27]}$ In the current study, we also found that the NSCLC patients with EGFR muta- 
Table 2: Clinical features of 39 adenocarcinoma patients of lung treated with EGFR-TKI

\begin{tabular}{|c|c|c|c|c|c|c|c|c|c|c|}
\hline Gender & Age (years) & Smoking & Prior regimen $^{1}$ & TKI & Response & PFS (months) & OS (months) & Survival status ${ }^{2}$ & Mutation 1 & Mutation 2 \\
\hline $\bar{M}$ & 41 & $\mathrm{Y}$ & 3 & Erlotinib $^{3}$ & E-PR & 19.5 & 26.87 & $\mathrm{D}$ & delE746-A750 & \\
\hline M & 61 & $\mathrm{Y}$ & 0 & Gefitinib & E-PR & 5.37 & 8.6 & $\mathrm{D}$ & delE746-A750 & \\
\hline $\mathrm{F}$ & 50 & $\mathrm{~N}$ & 0 & Gefitinib & E-PR & 6.87 & 15.87 & $\mathrm{D}$ & delE746-A750 & \\
\hline M & 55 & $\mathrm{Y}$ & 1 & Erlotinib & E-PR & 5.4 & 9.87 & $\mathrm{D}$ & delE746-A750 & \\
\hline $\mathrm{F}$ & 79 & $\mathrm{~N}$ & 1 & Gefitinib & E-PR & 20.17 & 28.1 & $\mathrm{D}$ & delL747-P753 & \\
\hline $\mathrm{F}$ & 51 & $\mathrm{~N}$ & 4 & Erlotinib $^{3}$ & E-PR & 5.8 & 5.8 & $\mathrm{D}$ & delK745-A750 & \\
\hline M & 54 & $\mathrm{~N}$ & 1 & Gefitinib & E-PR & 5.93 & 17.73 & A & delL747-P753insS & \\
\hline $\mathrm{F}$ & 71 & $\mathrm{~N}$ & 0 & Gefitinib & E-PR & 5.37 & 18.3 & $\mathrm{D}$ & delE746-A750 & \\
\hline M & 69 & $\mathrm{Y}$ & 0 & Gefitinib & E-PR & 5.43 & 14.13 & $\mathrm{D}$ & delL747-E749 & A750P \\
\hline $\mathrm{F}$ & 70 & $\mathrm{~N}$ & 0 & Gefitinib & PR & 14.23 & 14.7 & A & delL747-P753insS & \\
\hline M & 54 & $\mathrm{~N}$ & 0 & Gefitinib & PR & 18.9 & 18.9 & A & delE746-S752insV & \\
\hline M & 40 & $\mathrm{Y}$ & 0 & Gefitinib & PR & 11.17 & 23.97 & $\mathrm{D}$ & delE746-A750 & \\
\hline $\mathrm{F}$ & 71 & $\mathrm{Y}$ & 0 & Gefitinib & SD & 4.1 & 6.8 & $\mathrm{D}$ & delE746-A750 & \\
\hline M & 71 & $\mathrm{~N}$ & 1 & Gefitinib & SD & 10.33 & 14.1 & $\mathrm{D}$ & delE746-A750 & \\
\hline $\mathrm{F}$ & 57 & $\mathrm{~N}$ & 0 & Gefitinib & $\mathrm{PD}$ & 0.47 & 20.33 & A & delE746-A750 & \\
\hline M & 52 & $\mathrm{~N}$ & 1 & Erlotinib & E-PR & 16.37 & 34.63 & $\mathrm{D}$ & L858R & \\
\hline M & 73 & $\mathrm{~N}$ & 0 & Gefitinib & E-PR & 8.1 & 14.7 & $\mathrm{D}$ & L858R & \\
\hline M & 62 & $\mathrm{~N}$ & 0 & Gefitinib & E-PR & 11 & 23.57 & $\mathrm{D}$ & L858R & \\
\hline $\mathrm{F}$ & 51 & $\mathrm{Y}$ & 0 & Gefitinib & E-PR & 32.23 & 32.23 & A & L858R & \\
\hline $\mathrm{F}$ & 74 & $\mathrm{~N}$ & 0 & Gefitinib & E-PR & 8.3 & 9.33 & $\mathrm{D}$ & L858R & \\
\hline $\mathrm{F}$ & 74 & $\mathrm{~N}$ & 0 & Gefitinib & E-PR & 6.83 & 6.83 & $\mathrm{D}$ & L858R & \\
\hline $\mathrm{F}$ & 73 & $\mathrm{~N}$ & 0 & Gefitinib & E-PR & 14.27 & 21.17 & A & L858R & \\
\hline $\mathrm{F}$ & 47 & $\mathrm{~N}$ & 0 & Gefitinib & E-PR & 4.5 & 6.5 & $\mathrm{D}$ & L858R & \\
\hline $\mathrm{F}$ & 89 & $\mathrm{~N}$ & 0 & Gefitinib & E-PR & 11.9 & 16.73 & A & L858R & \\
\hline M & 43 & $\mathrm{~N}$ & 0 & Gefitinib & E-PR & 5.97 & 9.03 & $\mathrm{D}$ & L858R & \\
\hline $\mathrm{F}$ & 57 & $\mathrm{Y}$ & 0 & Gefitinib & E-PR & 10.4 & 24.03 & A & L858R & S768I \\
\hline M & 78 & $\mathrm{Y}$ & 0 & Gefitinib & PR & 9.57 & 11.17 & D & L858R & \\
\hline $\mathrm{F}$ & 68 & $\mathrm{~N}$ & 0 & Gefitinib & PR & 12.37 & 27.33 & A & L858R & \\
\hline $\mathrm{F}$ & 44 & $\mathrm{~N}$ & 0 & Gefitinib & PR & 8.07 & 11.87 & A & L858R & \\
\hline M & 68 & $\mathrm{~N}$ & 0 & Gefitinib & PR & 9.5 & 9.5 & A & L858R & \\
\hline $\mathrm{F}$ & 52 & $\mathrm{~N}$ & 0 & Gefitinib & SD & 5.37 & 12.77 & $\mathrm{D}$ & L858R & \\
\hline M & 48 & $\mathrm{~N}$ & 0 & Gefitinib & $\mathrm{SD}$ & 4.13 & 6.37 & D & L858R & \\
\hline $\mathrm{F}$ & 66 & $\mathrm{~N}$ & 1 & Gefitinib & SD & 5.53 & 9.47 & $\mathrm{D}$ & L858R & \\
\hline $\mathrm{F}$ & 55 & $\mathrm{~N}$ & 2 & Gefitinib & SD & 4.33 & 10.1 & $\mathrm{D}$ & L858R & \\
\hline $\mathrm{F}$ & 38 & $\mathrm{~N}$ & 0 & Gefitinib & PD & 2.13 & 21.47 & A & L858R & \\
\hline M & 81 & $\mathrm{~N}$ & 0 & Gefitinib & PD & 1.97 & 4.9 & $\mathrm{D}$ & L858R & \\
\hline $\mathrm{F}$ & 64 & $\mathrm{~N}$ & 1 & Erlotinib & E-PR & 6.07 & 32.5 & $\mathrm{D}$ & E709K & G719A \\
\hline $\mathrm{F}$ & 57 & $\mathrm{~N}$ & 2 & Gefitinib & PR & 11.93 & 22.03 & $\mathrm{D}$ & G719C & S768I \\
\hline $\mathrm{F}$ & 66 & $\mathrm{~N}$ & 2 & Gefitinib & PD & 2.8 & 3.6 & $\mathrm{D}$ & P772_V774dup & \\
\hline
\end{tabular}

${ }^{1}$ Number of prior chemotherapy regimens; ${ }^{2}$ survival status by the time of last follow-up; ${ }^{3}$ erlotinib was their second targeted therapy. Abbreviations: M: Male; F: Female; TKI: Tyrosine kinase inhibitor; PFS: Progression-free survival; OS: Overall survival; PR: Partial response; E-PR: Partial response detected on Day 14; SD: Stable disease; PD: Progressive disease; D: Died; A: Alive

tions had a high response rate $(29 / 39,74.4 \%)$ to EGFR-TKI treatment. The efficacy is similar to that in the study series published previously. Among the 39 patients, 27 received gefitinib as the first-line therapy. The response rate, PFS, and OS showed no significant difference to the overall study.

On the other hand, although the response rate to EGFR-TKI treatment was high, some of the NSCLC patients with $E G F R$ mutations were still non-responsive to TKIs in all reported series. Thus, detection of EGFR mutation alone is not sufficient for physicians to determine the best therapy. Earlier determination of EGFR-TKI treatment response will still be very helpful for physicians to make appropriate decision for further treatment. Although the changes in FDG uptake by PET has been reported useful in predicting early treatment response results, ${ }^{[19,20]}$ the number of cases was quite small and whether it could correlate with the prognosis was still undetermined. In this study, we have demonstrated that a very high percentage $(21 / 29,72.4 \%)$ of patients with PR could have radiological response detected as early as 14 days after TKI treatment. Since TKI therapy fails in about $25 \%$ of patients with EGFR mutations, our study suggests that early $C T$ imaging may be of value in selecting appropriate patients 
Table 3: Drug toxicities by common toxicity criteria grade

\begin{tabular}{lcc}
\hline Adverse events & CTC grade 1-2 (\%) & CTC grade 3-4 (\%) \\
\hline Nonhematological & $8(20.5)$ & $2(5.1)$ \\
Paronychia & $12(30.7)$ & $1(2.6)$ \\
Diarrhea & $18(46.2)$ & $3(7.7)$ \\
Skin rash & $15(38.5)$ & $1(2.6)$ \\
Dry skin & $14(35.9)$ & $1(2.6)$ \\
Acne & $10(25.6)$ & $1(2.6)$ \\
Nausea & $4(10.3)$ & $1(2.6)$ \\
Vomiting & $5(12.8)$ & $1(2.6)$ \\
Edema & $7(17.9)$ & 0 \\
Keratitis & $8(20.5)$ & 0 \\
Fatigue & $3(7.7)$ & 0 \\
Neuropathy & $9(25.0)$ & 0 \\
Mucositis & $3(7.7)$ & 0 \\
Constipation & $1(2.6)$ & 0 \\
Alopecia & $2(5.1)$ & 0 \\
Folliculitis & & \\
Hematological & $4(10.3)$ & $3(7.7)$ \\
Leukocytopenia & $16(41.0)$ & $2(5.1)$ \\
Anemia & $4(10.3)$ & $2(5.1)$ \\
Thrombocytopenia & 0 & $4(10.3)$ \\
Neutropenia & $6(15.4)$ & $1(2.6)$ \\
Nephrotoxicity & $11(28.2)$ & $2(5.1)$ \\
Hepatotoxicity & axicity criteria & \\
\hline Abbreviation: CTC: Common & & 0
\end{tabular}

Abbreviation: CTC: Common toxicity criteria

with TKI therapy. On the other hand, there were still eight patients actually ending up with a PR $(8 / 18,44 \%)$. Thus, the standard CT scan study on Day 56 still could not be replaced for determination of treatment response.

Interestingly, the median PFS and OS of the patients without early CT response were both longer than of patients with early CT response (11.9 months vs. 8.1 months for PFS and 24 months vs. 18.3 months for OS), but the differences were statistically non-significant. Since the patient number in this trial was small, future prospective studies with a larger patient number are necessary to clarify whether there are significant differences in the survival between patients with and without early radiological response.

In conclusion, this prospective study demonstrated a high correlation (72.4\%) in the radiological response between early (Day 14) and regular evaluation time (Day $56)$, and a very high percentage $(21 / 29,72.4 \%)$ of early radiological response to EGFR-TKIs in NSCLC patients with EGFR mutations and PR, which would advocate early radiological examination for EGFR-TKI therapy in NSCLC patients. It can be very helpful for physicians to make appropriate decision for further treatment, even though it still cannot replace the standard CT scan study on Day 56.

\section{Acknowledgments}

This work was supported by grants from the Department of Health (DOH96-TD-D-113-040), National
Health Research Institutes (NHRI 96-A1-MG-PP-04, NHRI-A1-MG-98-PP-04) and National Science Council (NSC97-2320-B-400-006-MY3), Taiwan.

\section{REFERENCES}

1. Bach PB, Ginsberg RJ. Epidemiology of lung cancer. In Lung cancer. In: Ginsberg RJ, editor. Hamilton, London: BC Decker Inc; 2002. p. 1-3.

2. Gabrielson E. Worldwide trends in lung cancer pathology. Respirology 2006;11:533-8.

3. Shepherd FA, Rodrigues Pereira J, Ciuleanu T, Tan EH, Hirsh V, Thongprasert S, et al.; National Cancer Institute of Canada Clinical Trials Group. Erlotinib in previously treated non-small-cell lung cancer. N Engl J Med 2005;353:123-32.

4. Chang A, Parikh P, Thongprasert S, Tan EH, Perng RP, Ganzon D, et al. Gefitinib (IRESSA) in patients of Asian origin with refractory advanced non-small cell lung cancer: Subset analysis from the ISEL study. J Thorac Oncol 2006;1:847-55.

5. Fukuoka M, Yano S, Giaccone G, Tamura T, Nakagawa K, Douillard JY, et al. Multi-institutional randomized phase II trial of gefitinib for previously treated patients with advanced non-small-cell lung cancer (The IDEAL 1 Trial) [corrected]. J Clin Oncol 2003;21:2237-46.

6. Kris MG, Natale RB, Herbst RS, Lynch TJ Jr, Prager D, Belani CP, et al. Efficacy of gefitinib, an inhibitor of the epidermal growth factor receptor tyrosine kinase, in symptomatic patients with non-small cell lung cancer: A randomized trial. JAMA 2003;290:2149-58.

7. Lynch TJ, Bell DW, Sordella R, Gurubhagavatula S, Okimoto RA, Brannigan BW, et al. Activating mutations in the epidermal growth factor receptor underlying responsiveness of non-small-cell lung cancer to gefitin. N Engl J Med 2004;350:2129-39.

8. Paez JG, Jänne PA, Lee JC, Tracy S, Greulich H, Gabriel S, et al. EGFR Mutations in Lung Cancer: Correlation with Clinical Response to Gefitinib Therapy. Science 2004;304:1497-500.

9. Huang SF, Liu HP, Li LH, Ku YC, Fu YN, Tsai HY, et al. High frequency of epidermal growth factor receptor mutations with complex patterns in non-small cell lung cancers related to gefitinib responsiveness in Taiwan. Clin Cancer Res 2004;10:8195-203.

10. Kosaka T, Yatabe Y, Endoh H, Kuwano H, Takahashi T, Mitsudomi T. Mutations of the epidermal growth factor receptor gene in lung cancer: Biological and clinical implications. Cancer Res 2004;64:8919-23.

11. Mitsudomi T, Kosaka T, Endoh H, Horio Y, Hida T, Mori S, et al. Mutations of the epidermal growth factor receptor gene predict prolonged survival after gefitinib treatment in patients with non-small-cell lung cancer with postoperative recurrence. J Clin Oncol 2005;23:2513-20.

12. Han SW, Kim TY, Hwang PG, Jeong S, Kim J, Choi IS, et al. Predictive and prognostic impact of epidermal growth factor receptor mutation in non-small-cell lung cancer patients treated with gefitinib. J Clin Oncol 2005;23:2493-501.

13. Asahina H, Yamazaki K, Kinoshita I, Sukoh N, Harada M, Yokouchi $\mathrm{H}$, et al. A phase II trial of gefitinib as first-line therapy for advanced non-small cell lung cancer with epidermal growth factor receptor mutations. Br J Cancer 2006;95:998-1004.

14. Inoue A, Suzuki T, Fukuhara T, Maemondo M, Kimura Y, Morikawa N, et al. Prospective phase II study of gefitinib for 
chemotherapy-naive patients with advanced non-small-cell lung cancer with epidermal growth factor receptor gene mutations. J Clin Oncol 2006;24:3340-6.

15. Tamura K, Okamoto I, Kashii T, Negoro S, Hirashima T, Kudoh S, et al. West Japan Thoracic Oncology Group. Multicentre prospective phase II trial of gefitinib for advanced non-small cell lung cancer with epidermal growth factor receptor mutations: Results of the West Japan Thoracic Oncology Group trial (WJTOG0403). Br J Cancer 2008;98:907-14.

16. Yang CH, Yu CJ, Shih JY, Chang YC, Hu FC, Tsai MC, et al. Specific EGFR mutations predict treatment outcome of stage IIIB/IV patients with chemotherapy-naive non-small-cell lung cancer receiving first-line gefitinib monotherapy. J Clin Oncol 2008;26:2745-53.

17. Ettinger D. $2010 \mathrm{NCCN}$ guideline for NSCLC. Available from: http:// www.nccn.org/professionals/physician_gls/f_guidelines.asp. [Last accessed on 2010 July 15].

18. Chang A. 2009 NCCN Asia consensus statement. Available from: http://www.ncen-asia.org/Adaptation.aspx?CatorID=3 and $\mathrm{KoID}=4$. [Last accessed on 2010 July 15].

19. Sunaga N, Oriuchi N, Kaira K, Yanagitani N, Tomizawa Y, Hisada T, et al. Usefulness of FDG-PET for early prediction of the response to gefitinib in non-small cell lung cancer. Lung Cancer 2008;59:203-10.

20. Ullrich RT, Zander T, Neumaier B, Koker M, Shimamura T, Waerzeggers $\mathrm{Y}$, et al. Early detection of erlotinib treatment response in NSCLC by 3'-deoxy-3'-[F]-fluoro-L-thymidine ([F] FLT) positron emission tomography (PET). PLoS One 2008;3:e3908.

21. Tanvetyanon T, Eikman EA, Sommers E, Robinson L, Boulware D,
Bepler G. Computed tomography response, but not positron emission tomography scan response, predicts survival after neoadjuvant chemotherapy for resectable non-small-cell lung cancer. J Clin Oncol 2008;26:4610-6.

22. Therasse P, Arbuck SG, Eisenhauer EA, Wanders J, Kaplan RS, Rubinstein L, et al. New guidelines to evaluate the response to treatment in solid tumors. European Organization for Research and Treatment of Cancer, National Cancer Institute of the United States, National Cancer Institute of Canada. J Natl Cancer Inst 2000;92:205-16.

23. Hsieh MH, Fang YF, Chang WC, Kuo HP, Lin SY, Liu HP, et al. Complex Mutation Patterns of Epidermal Growth Factor Receptor Gene Associated with Variable Responses to Gefitinib Treatment in Patients with Non-Small Cell Lung Cancer. Lung Cancer 2006;53:311-22.

24. Chang JW, Chou CL, Huang SF, Wang HM, Hsieh JJ, Hsu T, et al. Erlotinib response of EGFR-mutant gefitinib-resistant non-small-cell lung cancer. Lung Cancer 2007;58:414-7.

25. Mok TS, Wu YL, Thongprasert S, Yang CH, Chu DT, Saijo N, et al. Gefitinib or carboplatin-paclitaxel in pulmonary adenocarcinoma. N Engl J Med 2009;361:947-57.

26. Maemondo M, Inoue A, Kobayashi K, Sugawara S, Oizumi S, Isobe $\mathrm{H}$, et al. Gefitinib or chemotherapy for non-small-cell lung cancer with mutated EGFR. N Engl J Med 2010;362:2380-8.

27. Yoshida K, Yatabe Y, Park JY, Shimizu J, Horio Y, Matsuo K, et al. Prospective validation for prediction of gefitinib sensitivity by epidermal growth factor receptor gene mutation in patients with non-small cell lung cancer. J Thorac Oncol 2007;2:22-8. 